Federica Rovelli

\title{
Die Skizzenbuch-Ausgaben und ihre mögliche digitale Zukunft
}

Welchen Status `Skizzenbuch-Ausgaben ‘ besitzen, ist schwer zu definieren. Allein beim Gedanken an die Beethoven-Forschung, die sich als erste in der Musikwissenschaft diesem Bereich gewidmet hat, kann man feststellen, dass diese Bezeichnung bis heute sowohl Faksimile- als auch diplomatische und historisch-kritische Ausgaben - oder sogar eine Mischung all dieser verschiedenen Editionstypen-umfasst. ${ }^{\mathrm{I}}$ Solche Editionen stehen übrigens in einer langen Tradition, die mehr als hundert Jahre zurückreicht, und die dabei entwickelten Methoden haben sich mit der Zeit so oft verändert, dass jede Edition - aus einer epistemologischen Perspektive betrachtet - quasi als Unikat zu verstehen ist. Einige Tendenzen und wiederkehrende Probleme sind aber klar erkennbar und als spezifisch für diese Ausgabentypen anzusehen. Die Entwicklung dieses besonderen editorischen Bereichs im digitalen Zeitalter wird ähnliche Schwierigkeiten in den Griff bekommen müssen, wenngleich ihr mächtigere >Waffen`zur Verfügung stehen. Dieser Beitrag wendet sich daher in einer einleitenden Betrachtung zunächst den in den traditionellen Papier-Ausgaben erkennbaren konzeptionellen Problemen zu.

Transkriptionsmethode und >epistemologische Kurzschlüsse` In ihrer heute üblicheren Konzeption sind Editionen von Beethovens Skizzenbüchern grundsätzlich Quelleneditionen, die durch Faksimile, Transkription und kritischen Kommentar konstituiert sind. Die Transkriptionen bilden den Kern der Ausgabe: Ihre Ansätze prägen auffällig das Konzept der vollständigen Edition. Auch wenn nicht immer jede Phase von den anderen trennbar ist, durchläuft die Erschließung der Skizzentexte, grob vereinfacht, drei Stadien: Grundlegend ist eine befundbasierte Entzifferungsphase mit dem Hauptziel der Lesbar-

1 Zusammenfassend können die folgenden Ausgaben als Beispiele der unterschiedlichen Ansätze gelten: Joseph Schmidt-Görg: Beethoven, Drei Skizzenbücher zur Missa Solemnis. I Ein Skizzenbuch aus den Jahren I8I9/20, Bonn 1952 (diplomatische Transkription); Joseph Kerman: Beethoven. Autograph Miscellany from circa I786 to I799. British Museum Additional Manuscript 298or, ff.39-I62 (The »Kafka sketchbook«), London I970 (interpretierende Transkription); Sieghard Brandenburg: Keßlersches Skizzenbuch. Ludwig van Beethoven, Bonn I976-I978 (Beethoven. Skizzen und Entwürfe, Bd. 5) (linearisierte Transkription); William Kinderman: Artaria I95. Beethoven's Sketchbook for the »Missa solemnis« and the Piano sonata in E Major, opus Io9, Urbana/Chicago 2003 (diplomatisch-interpretierende Transkription). Die Entwicklung dieser Editionstypen wurde aus einer historischen Perspektive bereits beschrieben, vgl. dazu Federica Rovelli: Skizzeneditionen zu Beethoven. Nutzen und Aufgaben im wissenschaftsgeschichtlichen Rückblick, in: Vom Nutzen der Edition. Zur Bedeutung moderner Editorik für die Erforschung von Literatur- und Kulturgeschichte, hg. von Thomas Bein, Berlin/Boston 2015 (Beihefte zu Editio, Bd.39), S. 297-3II. 
keit (also der Überwindung der Lese-Schwierigkeiten, die besonders bei Beethovens Handschrift auftreten); darauf folgt eine interpretierende Phase (in der die musikalische Bedeutung des transkribierten Textes erschlossen wird); dann folgt eine dritte, kontexterschließende Phase, bei der jedes einzelne Notat in eine sowohl diachronische als auch synchronische Beziehung zum Kontext gestellt wird.

Die Entzifferungsphase ist im Zusammenhang mit den Modernisierungs- und Standardisierungsmaßnahmen zu sehen, die in allen Transkriptionen üblicherweise vorgenommen werden - und die jeweils historische und individuelle grafische Merkmale der Schrift modifizieren. Alle Besonderheiten im Duktus (Zeichengröße und -form der Notationssymbole, Neigungswinkel der Schriftzüge, wechselnde Federstärke et cetera) und Farben des Schreibmittels (oder wechselnde Sättigungsgrade der Tinte) werden in dieser Transkriptionsphase normalerweise standardisiert. Flüchtige oder unklare Zeichenpositionen, die oft in Skizzen zu finden sind, werden auf die gleiche Art und Weise behandelt; der Ansatz der Notenhälse am Notenkopf (rechts oder links) wird sowieso immer der heutigen Nutzung gemäß modernisiert. Diese erste Phase spielte in der Geschichte der Skizzeneditionen von Anfang an eine große Rolle: In den sogenannt diplomatischen< Transkriptionen wurden zum Beispiel ausschließlich solche editorischen Maßnahmen vorgenommenen, die das Hauptziel verfolgten, die Leseprobleme des Nutzers zu lösen. Dass solche Maßnahmen auch Informationsverluste oder Textverfälschungen generieren können, wurde normalerweise stillschweigend akzeptiert und nicht weiter diskutiert.

In der zweiten Phase werden Ergänzungsmaßnahmen vorgenommen, wodurch vor allem Schlüssel, Akzidenzien und Taktstriche hinzugefügt werden können. Die Notwendigkeit dieser Ergänzungsmaßnahmen ist in der Geschichte der Beethoven-Skizzeneditionen seit langer Zeit anerkannt. ${ }^{2}$ Die methodische Notwendigkeit dieser Interpretationsmaßnahmen ergibt sich aus dem Textstatus von Skizzen, und zwar aus deren spezieller Adressierung: Skizzen - im Unterschied zu jeder anderen Textsorte - sind immer selbstadressiert, Sender und Empfänger der textlichen Botschaft sind also identisch. Der Komponist, der für sich selbst schreibt und für keinen Kopisten salles klar und sauber niederzuschreiben braucht, benutzt viele Abkürzungsstrategien, um Zeit, Papier und Tinte zu sparen. Viele Notationsdetails, die einfach zu erinnern oder aus dem Kontext und anderen Quellen erschließbar sind, werden aus schreibökonomischen Gründen stenografisch fixiert oder sogar weggelassen. Skizzentexte sind also definitionsgemäß unvollständig und fragmentarisch. Wenngleich die Skizzen in einer abgekürzten oder unvollständigen Form vorliegen, so schrieb der Komponist dennoch etwas nieder, was mit Sicherheit eine musikalische Bedeutung hatte. Die Interpretation beziehungs- 
weise der ergänzende Eingriff des Herausgebers ist deshalb ständig notwendig und als Konstruktionsarbeit zu verstehen (es muss betont werden, dass vom Herausgeber eine echte >Konstruktionsarbeit ‘ geleistet wird, weil kein zerstörter oder durch Überlieferung manipulierter Text zu rekonstruieren ist). ${ }^{3}$ Derselbe Herausgeber-Eingriff stellt aber gleichzeitig ein methodisches Problem dar, weil dadurch der besondere Textstatus von Skizzen - definitionsgemäß unvollständig und fragmentarisch - unvermeidbar verraten wird. Das Risiko einer echten Textverfälschung wird dann als Konsequenz immer größer und das Problem der Grenzen dieser verstärkt interpretierenden Etappe sehr akut. 4

In der dritten Phase wird die Verbindung der verschiedenen Notate geklärt, sodass sie im Mikro- und Makro-Kontext betrachtet werden können (mit >Mikro-Kontext $<$ und >Makro-Kontext $<$ ist jeweils die quelleninterne und die quellenübergreifende Ebene gemeint). Eine diachronische Kontextualisierung ermittelt auf der einen Seite die zeitliche Abfolge der Notate, also die Chronologie des Schreibprozesses. Eine synchronische Kontextualisierung erlaubt auf der anderen Seite, die topografischen Beziehungen der Notate zu deuten: Dadurch wird kenntlich gemacht, wenn der Komponist zum Beispiel verschiedene Varianten als mögliche gültige Alternativen (anders gesagt als »rivalisierende Varianten « $)^{5}$ niedergeschrieben hat oder wenn implizite Beziehungen zwischen Skizzen bestehen. Die letzten erwähnten synchronischen Beziehungen wurden schon in Papier-Skizzeneditionen betrachtet und kommentiert. Das Interesse für das Freilegen der Schreibprozesse - also für die `diachronische Kontextualisierung - kam dagegen erst in den I96oer-Jahren (mit den ersten Schritten der französischen critique génétique) auf und wurde bis heute von Skizzeneditionen nur teilweise behandelt. ${ }^{6}$ Die Deutung der

Gut bekannt sind die Kritiken, die in den I960er-Jahren von der amerikanischen Skizzenforschung (erstmals von Douglas Johnson und Lewis Lockwood) zur deutschen Reihe des Beethoven-Hauses (mit diplomatischen Übertragungen und unter Joseph Schmidt-Görg entstanden) geäußert wurden; vgl. dazu insbes. Lewis Lockwood: Ludwig van Beethoven. Ein Skizzenbuch zur Pastoralsymphonie op. 68 und zu den Trios op.70, I und 2. Vollständige, mit einer Einleitung und Anmerkungen versehene Ausgabe von Dagmar Weise, in: The Musical Quarterly 53 (1967), S. I28-r36; und Philip Gossett: Beethoven's Sixth Symphony. Sketches for the First Movement, in: Journal of American Musicological Societp 27 (I974), S. 248-280.

4 Einige Beispiele zum Problem der Textverfälschung als Konsequenz von Standardisierungen und Ergänzungsmaßnahmen werden besprochen bei Federica Rovelli: Epistemologia e fenomenologia delle edizioni dei quaderni di schizzi di Beethoven, in: Philomusica On-line I4/I (2015), S. 289-308.

5 Die Definition stammt aus Bernhard R. Appel: Variatio delectat - Variatio perturbat. Anmerkungen zu Varianten in der Musik, in: Varianten - Variants - Variantes, hg. von Christa Jansohn und Bodo Plachta, Tübingen 2005 (Beihefte zu Editio, Bd. 22), S. 7-24, hier S. 20.

6 Sieghard Brandenburg hat das Problem in seiner Edition als erster thematisiert; vgl. ders.: Keßlersches Skizzenbuch. Ludwig van Beethoven. Eine Seite der erwähnten Edition wird in den nächsten Beispielen ausführlicher kommentiert. 


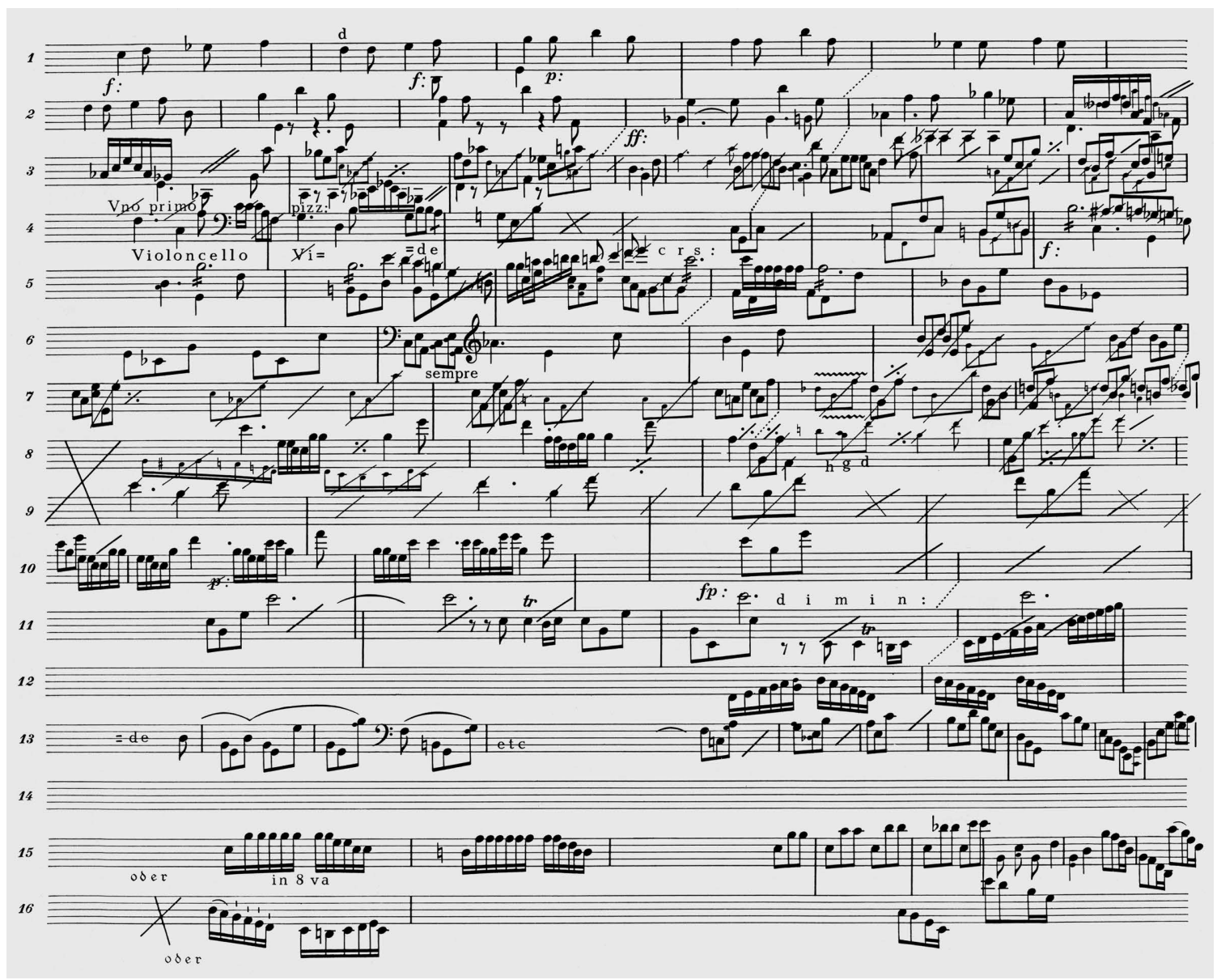

A в в I LD U N g 1 Ein Skizzenbuch zur Pastoralsymphonie op. 68 und zu den Trios op. 70, 1 und 2, hg. von Dagmar von Busch-Weise, Bonn 1961, S. 96

diachronischen Beziehungen, die die zeitliche Dimension erschließt, in der sich die Schaffensweise des Komponisten entwickelt hat, lässt also neue, für die SkizzenbuchAusgaben entscheidende methodologische Anstöße aufkommen, die weiter unten ausführlicher besprochen werden.7 Kontextualisierung heißt auf einer Makroebene aber auch, die Notate entsprechenden Werktexten zuzuordnen. Diese letzte Aufgabe des Herausgebers verwirklicht sich normalerweise in den Kommentartexten und Inhaltsverzeichnissen, aber auch durch Legende, Taktzähler und andere Hinweise, die direkt in der Transkription hinzugefügt werden können.

$7 \quad$ In diese Richtung bewegen sich auch folgende Publikationen Bernhard R. Appels: Zum Textstatus von Kompositions-Skizzen und -Entwürfen, in: Jahrbuch des Staatlichen Instituts für MusikforschungPreußischer Kulturbesitz, Stuttgart/Weimar 1999, S. I77-210; Über die allmähliche Verfertigung musikalischer Gedanken beim Schreiben, in: Die Musikforschung 56 (2003), S.347-365; Sechs Thesen zur genetischen Kritik kompositorischer Prozesse, in: Musiktheorie 20 (2005), S. II2-I22. 


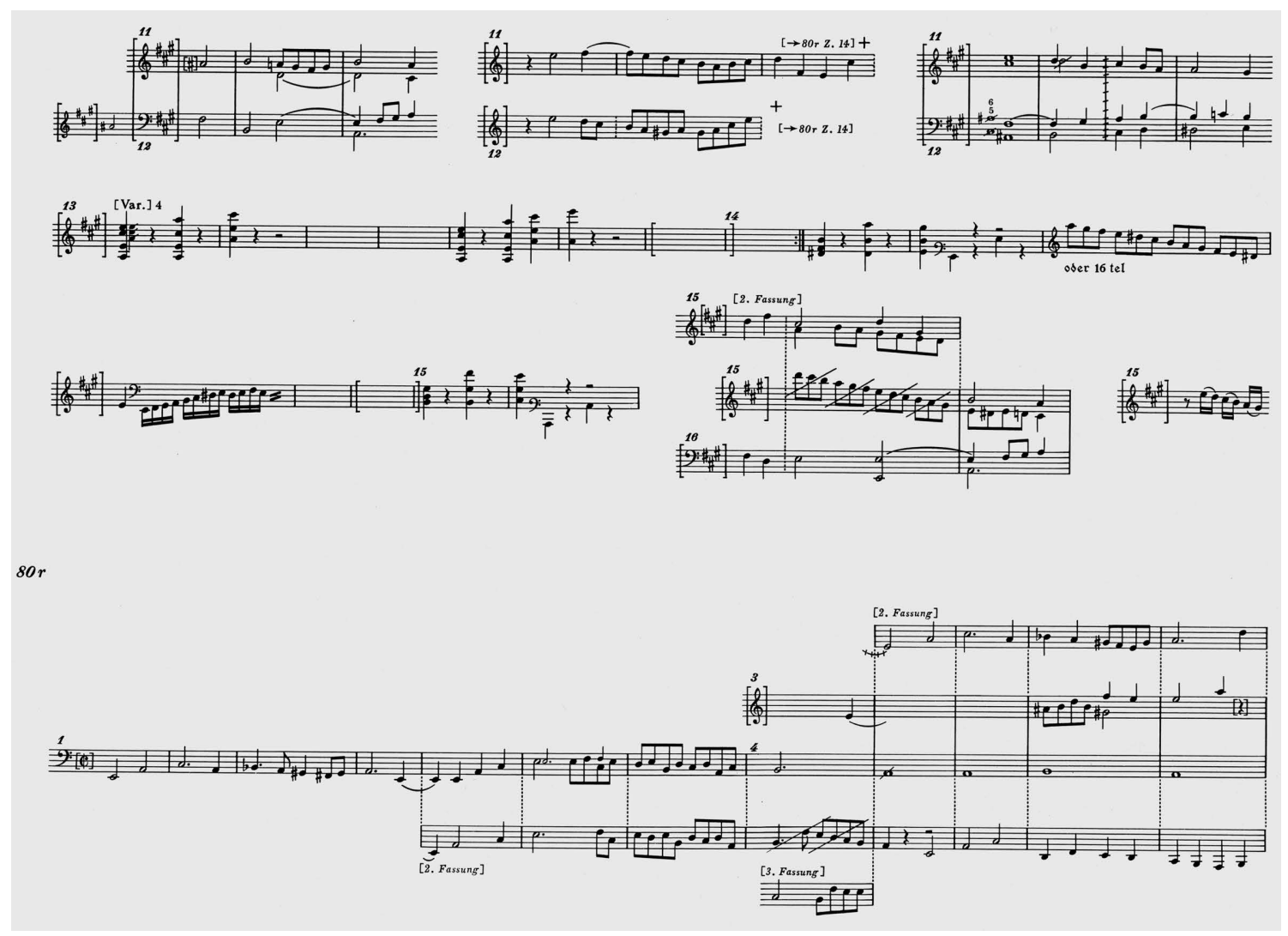

A B B I L D U N G 2 Ludwig van Beethoven. Keßlersches Skizzenbuch, hg. von Sieghard Brandenburg, Bonn 1978, Bd.1, S. 174

Diplomatisch oder linearisiert? Die Herausforderung dieser besonderen Editionsform besteht also, pragmatisch gesehen, in der Menge der editorischen Eingriffe, die wegen der Komplexität der Skizzen-Materialien in Frage kommen. Dadurch ergeben sich oft Argumentationskreise, echte unglückliche Vermischungen oder sogar editorische Inkonsistenzen (metaphorisch gesagt `epistemologische Kurzschlüsse`), die nicht immer auszugleichen sind. Man möchte zum Beispiel den definitionsgemäß >defizitären`Charakter der Skizzen nicht entstellen, kann als Herausgeber aber auch nicht die eigene Vermittlerrolle gegenüber dem Nutzer aufgeben. Und umgekehrt: Man möchte dem Nutzer soweit wie möglich helfen und ihm die lückenhafte, für Skizzen typische Notationsform zugänglich machen, kann aber weder editorische Ergänzungen hinzufügen noch die oben beschriebenen diachronischen Kontextualisierungen deutlich zum Ausdruck bringen, ohne die Topografie der Seite definitiv zu verändern und so den Anspruch auf authentisches Aussehen der Quelle aufzugeben. Als exemplarisch für derartige Widersprüche wird hier ein Problem diskutiert, das sich durch den Vergleich zweier unterschiedlicher Transkriptionen veranschaulichen lässt (Abbildung I und 2). 
In Abbildung I wird eine der ersten sogenannt >diplomatischen Goer-Jahre reproduziert. Das verfolgte Prinzip der »treuen Wiedergabe« ist deutlich und wird sogar im Vorwort jedes Bandes der diplomatischen Reihe ausführlich kommentiert. Die Transkriptionen wurden als Abschriften angefertigt, "gemäß der Vorlage seiten- und zeilengetreu«. Solche Abschriften wurden dann mit Hilfe einer Reproduktion des Manuskripts auf besonderes Cellophanpapier übertragen, »so daß jede Note und jedes Zeichen genau an die Stelle« kam, wo es sich im Original befand. ${ }^{8}$ In solchen Transkriptionen sind keine Schlüssel oder Vorzeichen ergänzt und es wird kein Kontextualisierungsversuch unternommen. Die Topografie der Notate ist aber durch den diplomatischen Ansatz automatisch erhalten, was dem Nutzer die Möglichkeit lässt, sich selbständig einer synchronischen Kontextualisierung anzunähern.

Ganz anders ist die von Sieghard Brandenburg angebotene Transkription des Keßler-Skizzenbuchs (Abbildung 2), deren Richtlinien auch von Clemens Brenneis und William Drabkin (jeweils in den Ausgaben der sogenannten Skizzenbücher Landsberg 5 und Artaria I97) übernommen wurden. ${ }^{9}$ Brandenburg bemühte sich vor allem, das Problem der sich überlappenden Schreibschichten durch eine >linearisierte Transkription zu lösen. ${ }^{\text {IO }}$ Die verschiedenen Schreibschichten, durch die Beethoven mehrere Varianten erzeugte, werden deswegen in seiner Ausgabe isoliert wiedergegeben. Die von Brandenburg vorgenommene editorische Maßnahme wirkt also als diachronische Kontextualisierung. Das von ihm erfundene Konzept erlaubt dem Nutzer, genetische Informationen unmittelbar und intuitiv zu erkennen: Alle Varianten sind klar auf einer sparadigmatischen Achse wiedergegeben, die durch die reine grafisch-synoptische Orientierung von oben nach unten - eine bestimmte Chronologie suggeriert. Die Nachteile dieser Transkriptionsmethode sind aber offensichtlich: Die Topografie der Seite kann nicht mehr beibehalten werden, dem Nutzer wird also nicht nur die Möglichkeit genommen, synchronische Kontextualisierungen selbst zu rekonstruieren, sondern es stellt sich auch wieder das Problem, sich zwischen Transkription und Faksimile hin und her bewegen

8 Vgl. zum Beispiel Schmidt-Görg: Beethoven, Drei Skizzenbücher zur Missa Solemnis, S. 9.

9 Clemens Brenneis: Ludwig van Beethoven. Ein Skizzenbuch aus dem Jahr I8o9 (Landsberg 5), Bonn I992/93 (Beethoven. Skizzen und Entwürfe, Bd. 7); William Drabkin: Ludwig van Beethoven. A Sketchbook from the Year IS2I (Artaria I97), Bonn 20Io (Beethoven. Skizzen und Entwürfe, Bd. 8).

Siehe dazu die Definition von Grésillon: »maschinenschriftliche Wiedergabe einer Handschrift, die alle Elemente des Originals überträgt, jedoch ohne sich an die Topographie der Seite zu halten. An deren Stelle tritt häufig eine Chronologisierung der Schreibelemente. Damit ist der erste Schritt in Richtung Interpretation getan, denn die Vertikalität der Umformulierungsparadigmen wird auf horizontal-syntagmatische Sequenzen übertragen, und dies ist nur möglich, indem räumlich Indizien in zeitliche Kriterien, d.h. in Aussagen über die Genese des Werkes überführt werden.« Almuth Grésillon: Literarische Handschriften. Einführung in die »critique génétique«, Bern u. a. I999 (Arbeiten zur Editionswissenschaft, Bd.4), S. 296. 
zu müssen. Ohne eine topografische Wiedergabe der Seiten wird dann die Trennung der Notierungen, die zum Beispiel nebeneinander auf der gleichen Zeile niedergeschrieben sind und in keiner klaren Verbindung stehen, grundsätzlich willkürlich. Ob es sich wirklich um eine einzige kontinuierliche, aber lückenhafte Notierung oder mehrere richtig getrennte Skizzen handelt, kann man nur selten feststellen. Trotzdem ist der Herausgeber durch die >Linearisierung $<$ der Transkription gezwungen, dem Leser eine klare Trennung zu suggerieren.

Zusammenfassend lässt sich sagen: Der Herausgeber von Skizzenbuch-Ausgaben befindet sich ständig in solchen Situationen. Er entwickelt ein editorisches Konzept, muss dann aber feststellen, dass sich im Laufe der Arbeit neue, am Anfang ungeahnte Probleme ergeben. Solche >Schachmatt<-Umstände - es lohnt sich, dies hier erneut zu betonen - offenbaren sich aufgrund der Komplexität der herausgegebenen Materialien.

Fragmentarischer Zustand (kubiert) Ein weiteres Problem der Skizzenbuch-Ausgaben, teilweise mit der schon besprochenen Frage der Makro-Kontextualisierung verbunden, hat mit dem fragmentarischen Zustand der Materialien zu tun. Auf diese Frage ging Brandenburg selbst ein, als er I99 über generelle Probleme der von ihm damals geleiteten Editionsarbeit sprach. Brandenburg sah die Hauptschwierigkeit der Skizzenforschung gerade im fragmentarischen Charakter der Skizzenmaterialien. ${ }^{\text {II }}$ Das Problem ist aber auf mehreren Ebenen angesiedelt und scheint sogar komplizierter als vom Forscher damals dargestellt. Man kann insgesamt drei Ebenen des Problems erkennen. Erstens: Die Skizzen selbst sind fragmentarisch. Sie brechen plötzlich ab und werden dann vielleicht auf einer anderen Seite weitergeführt. Manchmal geht es um eine direkte Fortsetzung, manchmal nicht, sodass einige Takte (wie viele kann man selten feststellen) übersprungen werden. $\mathrm{Ob}$ eine Notierung vom Komponisten als eine einzige unabhängige Einheit oder eher als Fortsetzung einer anderen verstanden worden ist, ist auch - wie oben schon erklärt - nicht trivial. Zweitens: Die Skizzenbücher sind ihrerseits (als kodikologische Einheiten) fragmentarisch überliefert worden. Was für eine Rolle einige unbedachte Sammler gespielt haben, ist gut bekannt, ${ }^{\mathrm{I} 2}$ manchmal ist aber Beethoven

»Die Überfülle des Materials und paradoxerweise seine Lückenhaftigkeit, bilden die eigentlichen Probleme der Beethoven-Skizzenausgabe. Der Modus der Edition ist eher eine sekundäre Frage.» Sieghard Brandenburg: Beethovens Skizzen. Probleme der Edition, in: Die Musikforschung 44 (I99I), S. 346-355, hier S. 354. Die Diskussion über die Probleme der »materiellen Philologie«, angestoßen von Alan Tyson, Douglas Johnson und Robert Winter und exemplarisch gelöst in ihrer heute geradezu kanonischen Publikation The Beethoven Sketchbooks. History, Reconstruction, Inventory (Oxford I985) war offenbar noch aktuell.

12 Der Fall vom Ignaz Sauer ist besonders bekannt. Er erwarb das heute sogenannte Sauer-Skizzenbuch 
selbst für Manuskript-Umstrukturierungen verantwortlich. Einige Seiten solcher Werkstattmaterialien wurden von ihm zum Beispiel absichtlich herausgerissen und innerhalb neuer kodikologischer Einheiten >recyceltr. Als Konsequenz muss der/die Herausgeber/in sich gelegentlich zwischen unterschiedlichen Quellen bewegen, um den ursprünglichen Zustand der Hauptquelle zu rekonstruieren. Drittens: Dass jedes Skizzenbuch einen gewissen einheitlichen Zeitraum widerspiegeln kann, wie Gustav Nottebohm in seinen Aufsätzen dargelegt hat, ist leider nicht immer als gültige Voraussetzung anzunehmen. ${ }^{13}$ William Kindermans Ausgabe des Skizzenbuchs Artaria I95 zählt zum Beispiel weitere 28 mit dem Skizzenbuch verbundene Quellen, die Beethoven gleichzeitig

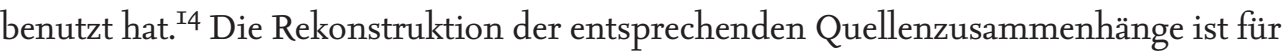
die Datierung der untersuchten Skizzenbücher besonders wichtig. Sie gelten dem Herausgeber als parallele Beweise, die entsprechend bewertet werden müssen. William Drabkin hat dann seinerseits in der Ausgabe des sogenannten Artaria r97 bewiesen, dass der Komponist dasselbe Skizzenbuch auch zu unterschiedlichen Zeitpunkten benutzte, sodass die vorhandenen Notate aus unterschiedlichen Jahren stammen können. ${ }^{15}$ Im Laufe der Zeit hat sich Beethovens Arbeitsweise so stark verändert, dass immer mehr Quellensorten (Schreibtischskizzenbücher, Taschenskizzenbücher und lose Blätter) gleichzeitig beziehungsweise parallel benutzt worden sind. Der fragmentarische Charakter der Materialien ist also insgesamt besonders markant und vonseiten der verantwortlichen Editionsleitung nicht nur schwer darzustellen, sondern auch als solcher nur mühsam in den Griff zu bekommen.

Zeitdimension und neue Philologien Das Interesse an der zeitlichen Dimension des Schreibens, also an der schon besprochenen diachronischen Mikro-Kontextualisierung nach Konzepten der französischen critique génétique, die heutzutage immer häufiger aufgegriffen werden, stellt ein weiteres gravierendes Problem der traditionellen, auf Papier konzipierten Skizzenbuch-Ausgaben dar. Statt eine methodische Diskussion darüber zu führen, soll im Folgenden ein konkretes Beispiel vorgestellt werden, das die Herausforderung der erwähnten textgenetischen Perspektive besser verdeutlichen kann (Abbildung 3).

bei der Auktion von Beethovens musikalischem Nachlass und zerlegte es in viele Einzelblätter, die er verschenkte oder verkaufte; vgl. Johnson/Tyson/Winter: The Beethoven Sketchbooks, S. II3-I23.

Die Idee ist als Hauptfaden in allen Publikationen Nottebohms erkennbar: Ein Skizzenbuch von Beethoven, hg. von Gustav Nottebohm, Leipzig I865; ders.: Beethoveniana. Aufsätze und Mittheilungen, Leipzig/Winterthur I872 (mit 29 schon in der Allgemeinen musikalische Zeitung veröffentlichten Beiträgen); ders.: Ein Skizzenbuch Beethovens aus dem Jahre I803, Leipzig I88o; Zweite Beethoveniana. Nachgelassene Aufsätze von Gustav Nottebohm, hg. von Eusebius Mandyczewski, Leipzig I887. 

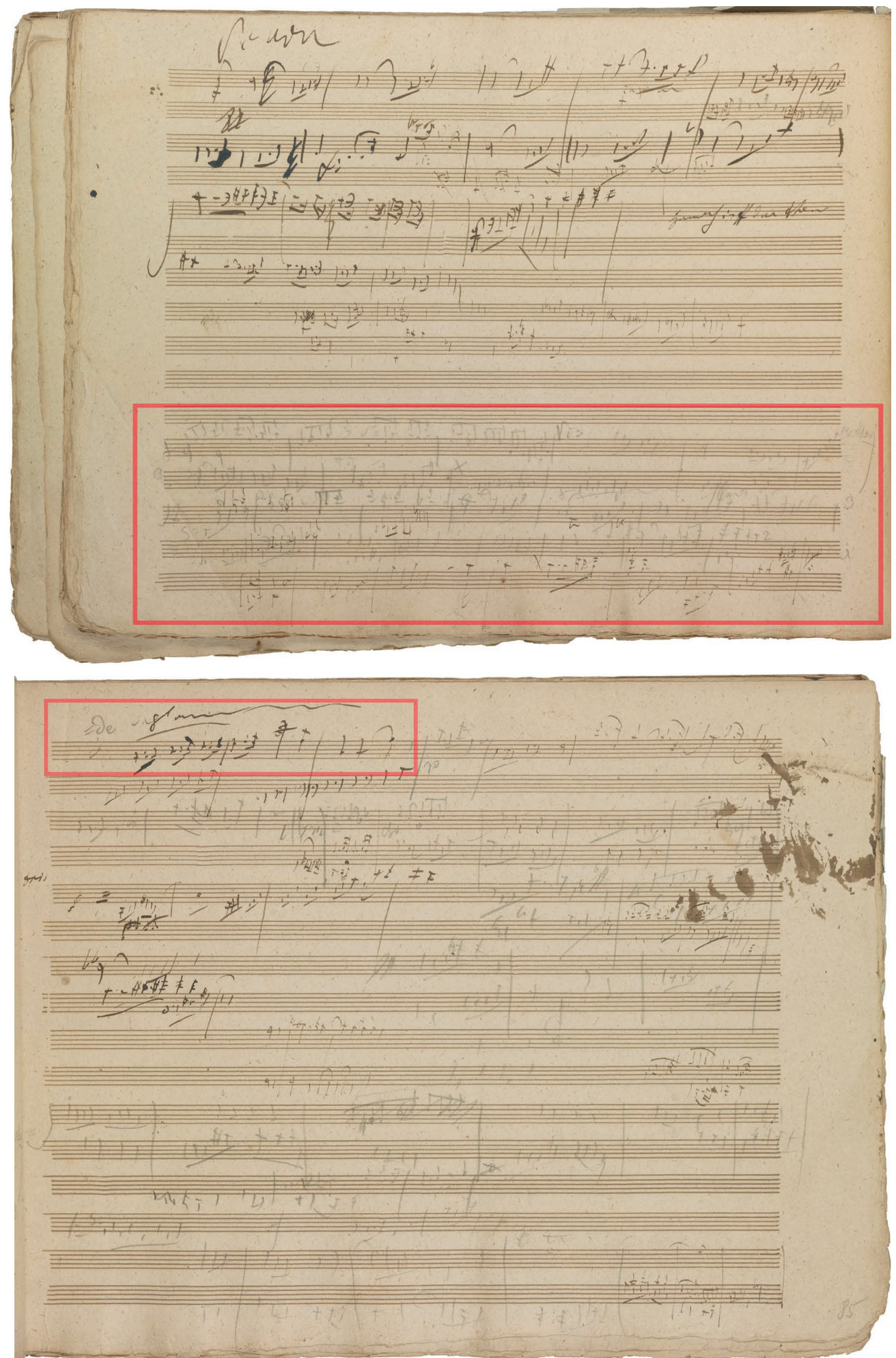

A в B I L D UN G 3 Beethoven: Entwurf zur Klaviersonate op. 101, Scheide-Skizzenbuch (The Scheide Library, Princeton University Library), S. 84 und 85 
Das Beispiel stammt aus dem von Beethoven in den Jahren I8I5/16 benutzten ScheideSkizzenbuch. ${ }^{16}$ Das Skizzenbuch enthält unter anderem einige Notate zur Klaviersonate op. Ior, insbesondere einen Entwurf, der sich auf zwei unterschiedlichen Seiten befindet. Die Notierung entspricht den Takten 9-46 des zweiten Satzes. Man kann Beethovens Schaffensweise im Fall dieser zwei Seiten auf Basis einiger skripturaler Merkmale rekonstruieren. Die Topografie der Seite, der Wechsel der verwendeten Schreibmittel, der Duktus der einzelnen Notate und die vorhandenen Verweiszeichen, die vom Komponisten benutzt worden sind, um die Verbindung seiner eigenen Skizzen klarer zu machen, sprechen für eine gewisse Reihenfolge der Schreiboperationen, die teilweise rekonstruiert werden kann. Der erste Takt auf Seite 85 ist ziemlich klar, mit einem sauberen Duktus und einer dunkleren Tinte geschrieben. Die gleiche dunklere Tinte ist auch für andere Notate auf den unteren Zeilen benutzt worden. Auf der gegenüberliegenden Seite 84 ist eine ähnliche Situation zu erkennen: Eine saubere Notierung - oben, wo das Wort »Vivace « zu lesen ist - wurde ebenfalls mit einer dunkleren Tinte niedergeschrieben. Beide Skizzen (also die Notate in dunkler Tinte) wurden höchstwahrscheinlich als erstes auf den noch leeren Seiten hinzugefügt: Beethoven schrieb ganz kurze Ideen nieder, die er später entwickeln wollte, und ließ Platz dazwischen, um dort direkt weitermachen zu können. Da er im folgenden Arbeitsschritt zum Bleistift griff, kann man in diesem Fall heute noch den Unterschied zwischen den früheren und den späteren Eintragungen erkennen. Auf dieser Basis kann man also vermuten, dass Beethoven am Anfang die erste Zeile auf Seite 85 nutzte, um nur drei Takte niederzuschreiben. Mit der Idee, an den vorhergehenden Takten zu arbeiten, entschied er sich, zusätzlich die noch leere Zeile auf dem unteren Rand der Seite 84 zu nutzen. Er war wahrscheinlich überzeugt, dass wenige Takte ausreichen würden, weshalb er in der letzten Zeile zu schreiben begann. Später bemerkte er aber, dass diese leere Zeile für die neu komponierten Takte nicht ausreichte und schrieb deshalb auf der darüber liegenden leer gebliebenen Zeile weiter, der vorletzten auf Seite 84, und so weiter bis hin zur fünftletzten Zeile. Um diese ungewöhnlichen Zeilenanschlüsse klarzustellen, fügte er mehrere Verweiszeichen hinzu (die Zahlen »I«, »«, »3« und zwei Kreuze). Die letzte Verbindung zu Seite 85 stellte er mit einem »Vi=de«Verweis her. Der ganze Entwurf auf Seite 84 ist deswegen von unten nach oben-entgegen der normalen Schreibpraxis - geschrieben und mit insgesamt zehn gekoppelten Verweiszeichen versehen worden.

»Der Text kommt mit dem Schreiben «, ${ }^{17}$ oder anders gesagt: Die Tätigkeit des Schreibens löst die kreative Schaffensfähigkeit aus. Das ist, kurz zusammengefasst, was

17 Almuth Grésillon: Über die allmähliche Verfertigung von Texten beim Schreiben, in: Schreiben als Kulturtechnik. Grundlagentexte, hg. von Sandro Zanetti, Berlin 2012, S. I52-I86, hier S. I52. 
dieses Beispiel beweist und nebenbei auch eine der Hauptthesen der critique génétique. Die damit verbundenen Überlegungen könnte man weiter in einer kognitivistischen Richtung entwickeln. Hier genügt zu klären, dass die bis heute benutzten und mit Papier-Medien verbundenen Transkriptionsmethoden nicht mehr als ein starres Transkriptionsprodukt anbieten könnten. Es gäbe keine Möglichkeit die Prozesshaftigkeit dieses Beispiels angemessen darzustellen, und dem Herausgeber würde nur der Weg der verbalen Beschreibung bleiben. Eine Ausgabe, die Skizzen und Entwürfe im Sinne der neuen methodischen Überlegungen der critique génétique erschließen möchte, sollte sich bemühen, eine >Redynamisierung < der auf Papier erstarrten Zeitdimension anzubieten. Sie sollte die Chronologie des Schreibprozesses so transparent wie möglich wiedergeben. Die traditionellen Papierdarstellungen stoßen damit aber an ihre `natürlichen Grenzen, wie Almuth Grésillon schon geklärt hat:

»Jeder Versuch, die Dreidimensionalität des textgenetischen Prozesses auf die Zweidimensionalität der Seite zu reduzieren, muß zwangsweise fehlschlagen [...]. Genau wie das Original selbst kann eine Transkription nur erstarrte Schreibspuren wiedergeben; also etwas Geschriebenes, nicht das Schreiben selbst. « ${ }^{\mathrm{I} 8}$

Mögliche Lösungen im Digitalen (das Projekt »Beethovens Werkstatt«) In einer Nachbemerkung des Jahres 2015 zu seinem Beitrag »Digitale Musikedition« hat Joachim Veit beschrieben, wie »die Euphorie der Anfangszeit digitaler Musikeditionen dem Bewusstsein von der Notwendigkeit einer kontinuierlichen Investition von Arbeitskraft [...] zur Fortführung des Begonnenen, speziell aber zur Umsetzung etlicher, im Arbeitsaufwand unterschätzter Konzepte gewichen ist. «I9 In der gleichen Nachbemerkung wird dann das Projekt »Beethovens Werkstatt. Genetische Textkritik und Digitale Musikedition«als einziges Vorhaben erwähnt, das sich mit textgenetischen Fragestellungen beschäftigt. Das damals noch junge Projekt hat inzwischen das zweite Modul seines Programms fast abgeschlossen und wird sich im vierten Modul einer vollständigen digitalen Edition eines Skizzenbuchs widmen. ${ }^{20}$ Im ersten Modul standen Variantenstellen in Beethovens Werkniederschriften im Zentrum der textgenetischen Analyse. Die dabei entwickelten Joachim Veit: Digitale Musikedition, in: Musikphilologie. Grundlagen - Methoden - Praxis, hg. von Bernhard R. Appel und Reinmar Emans, Laaber 20I7 (Kompendien Musik, Bd.3), S. 44-55, hier S. 53. Das Projekt wird für insgesamt I6 Jahre von der Akademie der Wissenschaften und der Literatur in Mainz gefördert. Wie der Untertitel»Genetische Textkritik und Digitale Musikedition« zeigt, werden dabei zwei Forschungsansätze miteinander verbunden. Diese wiederum korrespondieren mit den beiden Arbeitsstellen in Bonn (Beethoven-Haus) und Detmold (Hochschule für Musik - Universität Paderborn). Eine Beschreibung der einzelnen Module und die bis jetzt veröffentlichten Modell-Editionen sind auf der Webseite www.beethovens-werkstatt.de zu finden. Alle in diesem Beitrag erwähnten Weblinks sind über diese Homepage zu erreichen. 

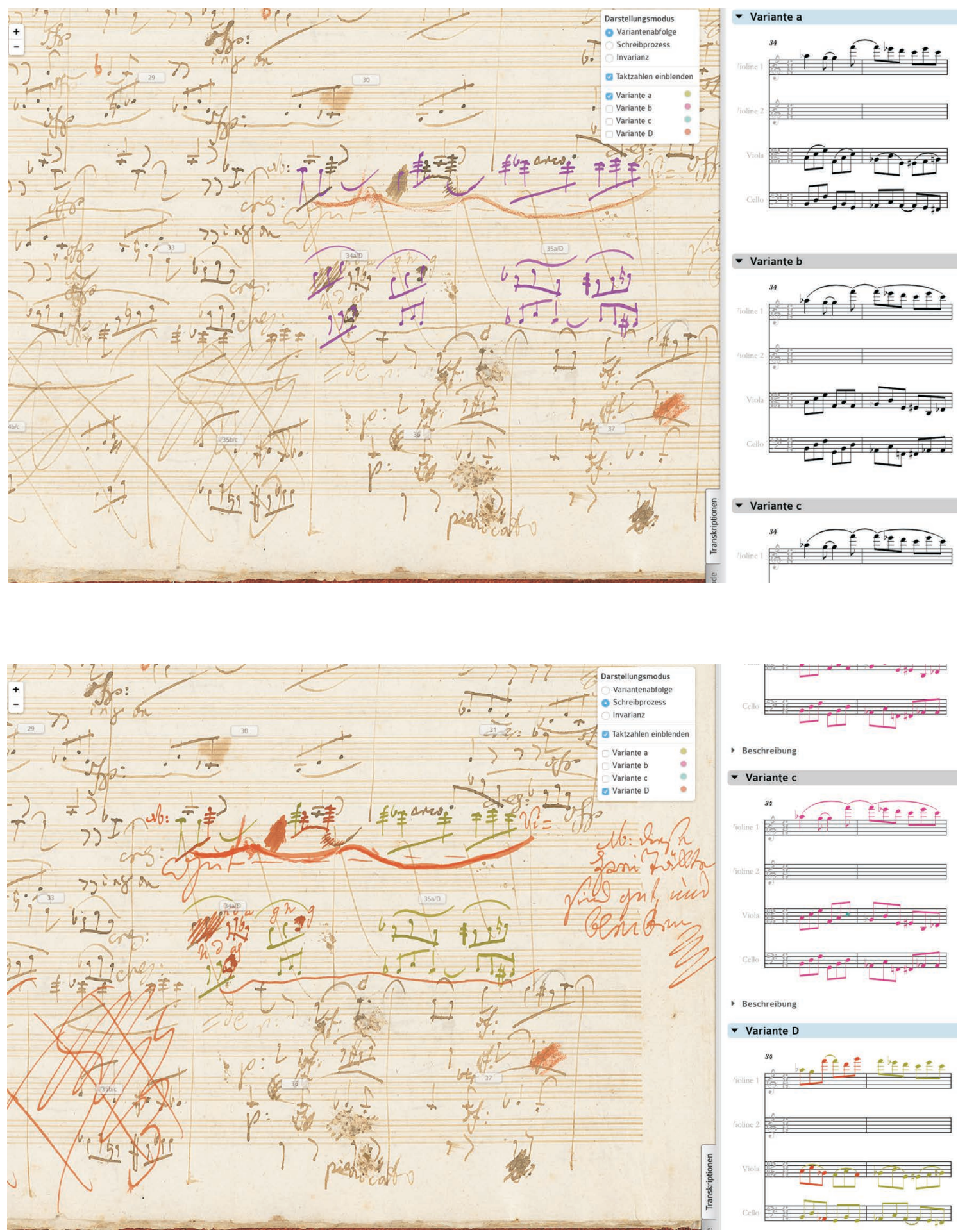

- Variante D

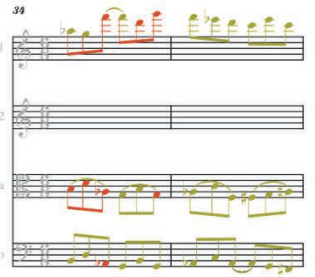



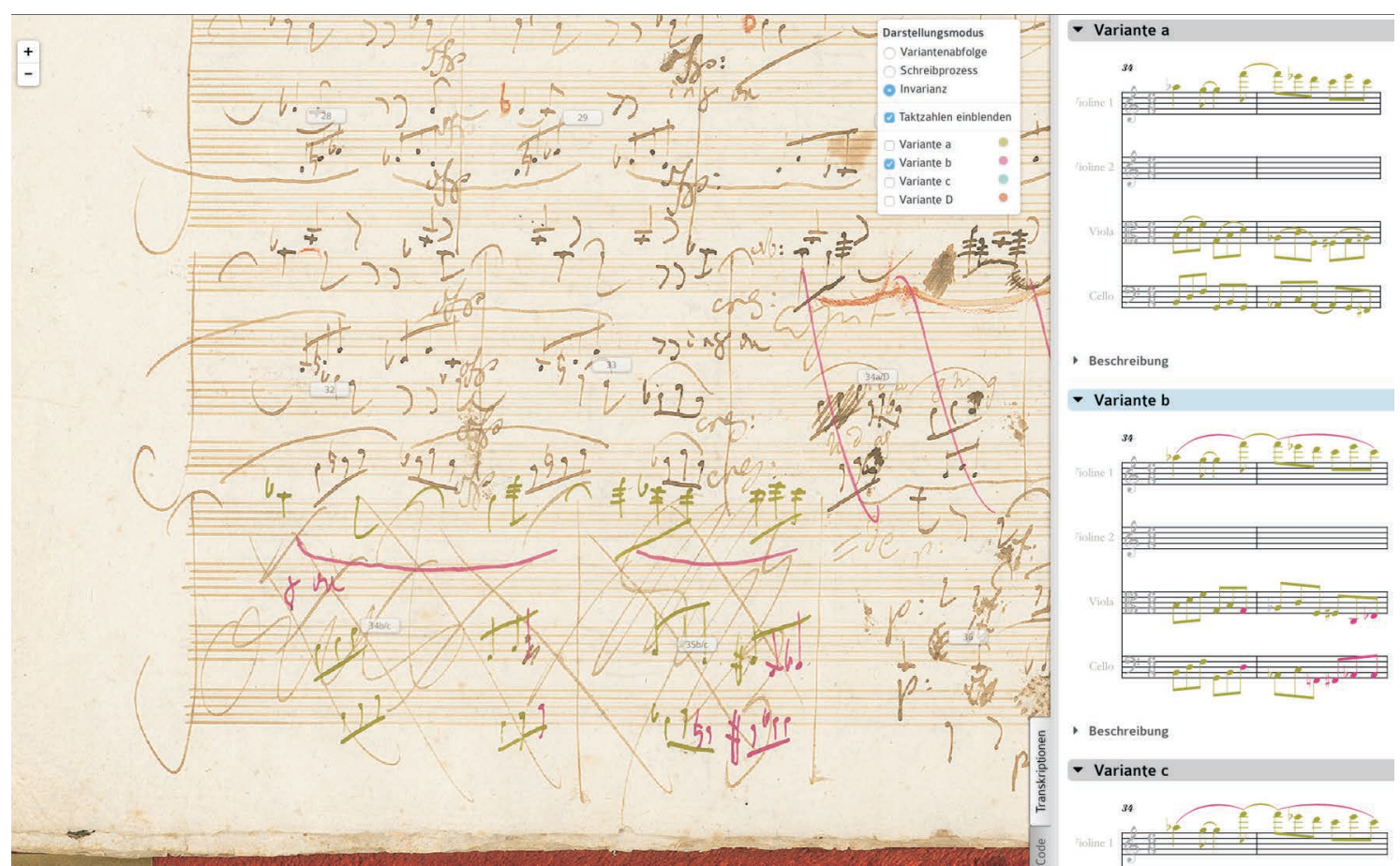

A в в ILDUNG 4 Screenshot aus der Modell-Edition von Ludwig van Beethoven: Streichquartett op. 59 Nr. 3 (2. Satz, Takt 34-35). Gegenüberliegende Seite oben: Hervorhebung von Variante a im Darstellungsmodus »Variantenabfolge«; darunter: Hervorhebung von Variante D im Darstellungsmodus »Schreibprozess«; oben: Hervorhebung von Variante b im Darstellungsmodus »Invarianz«

Vermittlungsstrategien bieten unterschiedliche Lösungen an, die auch im Fall der zukünftigen digitalen Skizzenbuch-Editionen eine große Rolle spielen werden und im Mittelpunkt dieses folgenden Abschnitts stehen. ${ }^{2 \mathrm{I}}$

Dass die Anwendung von digitalen Vermittlungsstrategien bei Skizzenbuch-Ausgaben besonders vielversprechend ist, wird anhand einer ersten Beobachtung sofort klar: Das für digitale Medien typische shypertextuelle Lektüreverhalten`stellt im Fall von Skizzenbuch-Editionen, wegen des beschriebenen fragmentarischen Zustands der Materialien, die ideale Lösung dar - wenn nicht sogar die einzig vernünftige. Wenn »ein nicht-lineares-Lesen, das Querverweisen nachgeht und eigene Pfade einschlägt « ${ }^{22}$ im Fall von historisch-kritischen Ausgaben ohnehin sinnvoll ist, ist solch »nicht-lineares-

21 Die nachstehenden Beobachtungen beschränken sich absichtlich auf eine konzeptionelle Ebene, um die Prinzipien solcher Vermittlungsstrategien zu erklären. Für Erklärungen, die mit technischen Aspekten verbunden sind, wird jeweils auf die Webseite des Projekts verwiesen.

22 Joachim Veit: Es bleibt nichts, wie es war. Wechselwirkungen zwischen Digitalen und »analogen« Editionen, in: Editio 24 (2010), S.37-52, hier S. 45. 
Lesen« für eine Skizzenbuch-Ausgabe umso mehr gerechtfertigt und sogar wünschenswert. Diese erste Beobachtung zeigt die Möglichkeiten digitaler Medien jedoch nur im Ansatz, reichen deren Fähigkeiten an Vermittlungsstrategien doch deutlich über Hypertextualität hinaus. Um die Frage ausführlicher zu betrachten, wird hier ein Beispiel des erwähnten Projekts »Beethovens Werkstatt« vorgestellt. Die Modell-Edition des Streichquartetts op. 59 Nr.3 (2. Satz, Takt 34-35) eignet sich insbesondere, einige vielversprechende Lösungen zu zeigen (Abbildung 4).

Die erwähnte Variantenstelle wurde wegen ihrer inhaltlichen Besonderheiten gewählt. Auf der Seite des im Beethoven-Haus Bonn befindlichen Arbeitsmanuskripts, ${ }^{23}$ der Werkniederschrift des dritten Razumouskp-Quartetts, sind insgesamt vier verschiedene Varianten niedergeschrieben, die als a, b, c und D bezeichnet werden. ${ }^{24}$ Die Varianten sind alle zwei Takte lang und unterscheiden sich durch einige Merkmale, die sowohl ihre skripturale Entstehung als auch ihren musikalischen Inhalt betreffen. Das Beispiel ist also äußerst vielschichtig und kann aus drei verschiedenen Perspektiven betrachtet werden. Auf einer ersten Ebene kann man feststellen, wie viele Varianten für Beethoven in Frage kamen, wie lang sie waren und wo genau sie auf der Manuskriptseite zu finden sind (was im Fall von Um- und Über-Schreibungen nicht so trivial ist, wie man vielleicht annehmen könnte). Auf der Ebene der reinen Skriptur kann man die von Beethoven benutzten Schreibstrategien betrachten beziehungsweise einzelne Schreibschichten isolieren, mit dem Ziel, ad-hoc- und Revisions-Varianten zu erkennen und, wo möglich, die Mikrochronologie des Schreibablaufs zu rekonstruieren. Ein Vergleich von Varianzrespektive Invarianz-Anteilen kann (drittens und endlich) erhellen, ${ }^{25}$ wo das kompositorische Problem lag, das Beethovens Überarbeitung seines ursprünglichen Textes ausgelöst hatte. Die Modell-Edition, exemplarisch für diese Variantenstelle konzipiert, fasst die drei erwähnten Perspektiven zusammen, isoliert sie aber gleichzeitig: Somit werden den Interessierten verschiedene Blickwinkel auf die untersuchte Textstelle angeboten, ohne dass diese einander überschneiden.

Die unterschiedlichen, isolierten Perspektiven (in Abbildung 4 als »Darstellungsmodus« gekennzeichnet) können einfach per Mausklick ausgewählt werden. Mit dem ersten »Darstellungsmodus« können die Nutzenden die Varianten-Reihenfolge inner-

D-BNba, BH 62.

Große respektive kleine Buchstaben benennen jeweils geschlossene respektive offene Varianten; vgl. dazu die Glossar-Definition des Projekts »Beethovens Werkstatt« auf der Seite http://beethovenswerkstatt.de/glossary/variante-2-o-o (2I. Februar 20I8).

Zum Begriff `Invarianz`vgl. Susanne Cox/Federica Rovelli: Invarianz - Ein Begriff zur genetischen Textkritik, in: Expertengespräch zur genetischen Textkritik im Bereich Musik, Mainz (2015), http://beethovens-werkstatt.de/prototyp/expertenkolloquium/invarianz-ein-begriff-zur-genetischen-textkritik (2I. Februar 2018). 
halb der Manuskriptseite beziehungsweise Faksimilereproduktion erkennen. Die Transkription der Varianten ist in Verbindung mit einer entsprechenden, auf dem Faksimile einblendbaren farblichen Hervorhebung abrufbar. Mit Hilfe farblicher Hervorhebungen wird nicht nur der Vergleich zwischen Manuskript und Transkription erheblich erleichtert, sondern auch jede editorische Maßnahme der Herausgebenden stransparentく. Mit dem zweiten Darstellungsmodus (»Schreibprozess«) wird den Nutzenden dann eine neue Perspektive eröffnet, in der die unterschiedlichen auf dem Manuskript erkennbaren Schreibschichten im Mittelpunkt stehen.

Durch diese zweite Perspektive kann man zum Beispiel verstehen, dass die letzte Variante D von Takt 34-35 durch eine besondere Schreibstrategie entstanden ist. Beethoven >recycelte $<$ in diesem Fall einen Teil der alten und schon gestrichenen Variante a, adaptierte einige ihrer Zeichen und fügte manche neue hinzu. Dass diese Variante D aus zwei kombinierten Schreibschichten gebildet ist, wird im Endeffekt durch die neue Einfärbung klar (vergleiche Abbildung 4): Die grünen und orangen Zeichen entsprechen jeweils der älteren beziehungsweise neueren Schreibschicht. Dank des letzten Darstellungsmodus »Invarianz « kann eine dritte Perspektive eingenommen werden, in der die Varianten durch eine weitere Einfärbungsfunktion auf einer inhaltlichen Ebene verglichen werden. Die Textsegmente, die zum Beispiel von Variante a zu Variante b unverändert geblieben sind, werden mit der gleichen Kolorierung markiert; der Varianzanteil ist also durch die abweichende Einfärbung mit bloßem Auge erkennbar.

Die im Quartett-Beispiel sallgegenwärtigen Hervorhebungen erfüllen eine deiktische Funktion - wenn auch mit unterschiedlichen Aufgaben. ${ }^{26}$ Sie stellen also eine Vermittlungsform dar, die gelegentlich auch in den traditionellen Papier-Ausgaben zu

26 Dazu wird eine MEI-Codierung in Verbindung mit Scalable Vector Graphics (svg) genutzt. Die einzelnen Zeichen des Manuskripts werden getrennt voneinander manuell mit einem Grafikbearbeitungsprogramm nachgezogen und gespeichert. An entsprechender Stelle in der Codierung wird jeder so erstellte Pfad mit dem entsprechenden Zeichen verknüpft. Als arbeitstechnisches Hilfsmittel wurde dazu im Projekt die sogenannte Genetic Sandbox entwickelt. Zu den oben genannten technischen Begriffen (MEI und svg) siehe die Definitionen des Projekts "Beethovens Werkstatt« auf der Glossar-Seite http://beethovens-werkstatt.de/technisches-glossar (2I. Februar 20I8). Eine Beschreibung und zwei konkrete Beispiele der Genetic Sandbox sind auf dieser Seite zu finden: http://beetho vens-werkstatt.de/genetic-sandbox (2I. Februar 20I8). Der Begriff $>$ Deixis $<$ (aus dem altgriechischen $=$ zeigen) wird in diesem Kontext im Sinne von Bernhard R. Appel verwendet. In seiner Definition, die bewusst von der Bedeutung abweicht, die in der Linguistik vorausgesetzt wird, bezieht sich das Wort auf eine Kommunikationsstrategie: In den Dramaturgietheorien können auch Gesten, etwa eine angehobene Hand, ein >deiktisches Element< sein und somit eine >deiktische Funktion besitzen; vgl. Bernhard R. Appel: Music as Composed Text. Reflections on the Content and Method of the »Critique Génétique« of Musical Works, in: Genèses musicales, hg. von Nicolas Donin, Almuth Grésillon und Jean-Louis Lebrave, Paris 20I5, S. 35-44, hier S. 43, Anm. I4. 
finden ist, ${ }^{27}$ deren Macht aber durch die digitalen Medien entscheidend potenziert wurde. Diese selbsterklärende Vermittlungsstrategie erlaubt, ein einzelnes Zeichen, aber auch Zeichen-Komplexe zweifelsfrei zu identifizieren, anders gesagt einen Befund und seine Deutung direkt miteinander zu verknüpfen. Die vom Herausgeber gesammelten Informationen sind durch solch eine »weitgehend non-verbale Darstellung [...] nicht nur visuell schnell zu erfassen, sondern auch für einen internationalen Nutzerkreis [...] leichter zu verstehen. ${ }^{28}$ Auf abstrahierter Ebene beschrieben: Das digitale Medium ermöglicht durch solche Maßnahmen, die ursprüngliche Komplexität der betrachteten Beispiele deutlich zu reduzieren. Das Prinzip kann bestimmt auch auf Skizzenbuch-Ausgaben angewendet werden, um zwischen einer grundlegend diplomatischen und einer stärker interpretierenden Transkription zu vermitteln. Zuerst wird sich aber die Frage stellen, ob das Problem der schnellen Vergleichbarkeit zwischen Transkription und Faksimilereproduktion im Digitalen noch besteht - also ob eine diplomatische Übertragung überhaupt noch als notwendig empfunden wird.

Eine letzte Anmerkung kann man hier auch bezüglich des Problems der Zeitdimension hinzufügen: Bei allen Perspektiven, die die Modell-Edition des Quartetts op. 59 Nr.3 bietet, wurde immer wieder eine statische Vorstellung des Textes benutzt (sowohl auf der Manuskript-, als auch auf der Transkriptions-Ebene). Auch wenn die Schreibprozesse dank der Einfärbung vermittelt werden, wird eine zeitliche Darstellung solcher Prozesse - also eine echte >Redynamisierung $<$ - nur suggeriert, aber noch nicht im eigentlichen Sinne visualisiert. Erst die Herstellung der sogenannten >Rekonstruktionsansicht $<$ hat das Zeigen von dynamischen Schreiboperationen ermöglicht. Dieses Werkzeug - immer auf der Basis der schon beschriebenen farblichen Hervorhebungen entwickelt - wurde innerhalb des Projekts »Beethovens Werkstatt«jüngst realisiert und ermöglicht es, die verschiedenen Schreibschichten allmählich, in der vom Herausgeber vermuteten Reihenfolge schrittweise anzuzeigen. ${ }^{29}$ Der Nutzer selbst kann per Klick die Schreibschichten ein- oder ausblenden, das Ergebnis kann dann eventuell in Form einer filmischen Animation gespeichert werden. Die Rekonstruktionsansicht kann also im Zusammen-

27 Siehe Ludwig van Beethoven. Sonate für Violoncello und Klavier op. 69, I. Satz [Faksimile-Ausgabe], hg. von Jens Dufner und Lewis Lockwood, Bonn 2015, S. 25, 27 und 29.

28 Anette Müller: Überlegungen zu einer digitalen Textgenetischen Darstellung von R. Schumanns op. 70, in: Digitale Editionen zwischen Experiment und Standardisierung. Musik - Text - Codierung, hg. von Peter Stadler und Joachim Veit, Tübingen 2009 (Beihefte zu Editio, Bd.3I), S.33-46, hier S. 35.

Die Beispiele von op. 75, Nr. 2 und op. 93 wurden mit einer solchen >Rekonstruktionsansicht $<$ versehen und sind jeweils auf diesen Seiten zu finden: http://nagano.upb.de:29999/75cr39af und http://nagano. upb.de:29999/f2da6d8r. Dazu wurden keinerlei Animationen hergestellt: Die notwendigen Informationen über die einzelnen >Etappen des Schreibens sind genau wie der Notentext durch MEI codiert. Die Codierung und die damit verbundenen SVG-Pfade ermöglichen es, den Prozess direkt zu visualisieren. 
hang mit der erwähnten Redynamisierungsfrage gute Lösungen anbieten und dementsprechend die methodische Anforderung einer »Simulation von Schreib- und Kompositionsabläufen « ${ }^{\circ}$ vonseiten der neuen Philologien befriedigen: Der Schreibprozess in den Skizzenbüchern könnte mit ähnlichen Mitteln rekonstruiert und so in seiner zeitlichen Dimension dargestellt werden. Eine zugespitzte Formulierung wäre in diesem Fall sogar akzeptabel und würde ein Plädoyer für digitale Skizzenbuch-Ausgaben rechtfertigen, weil nur »das digitale Medium [...] die im Schriftbild geronnene Zeitlichkeit kompositorischer Arbeitsprozesse in adäquater Weise aufgelöst darzustellen « vermag. ${ }^{3 \mathrm{I}}$

30 Hans Walter Gabler: Computergestütztes Edieren und Computer-Edition, in: Textgenetische Edition, hg. von Hans Zeller und Gunter Martens, Tübingen I998 (Beihefte zu Editio, Bd. ro), S.315-328, hier S.32I.

31 Veit: Es bleibt nichts, wie es war, S. 49 . 


\section{Inhalt}

Vorwort 8

INTERPRETATION - BEGRIFF, METHODE, PRAXIS

Laure Spaltenstein Interpretation als treue Übersetzung. Zur Frühgeschichte eines vieldeutigen Begriffs I5

Kai Köpp Von der Quelle zur Methode. Zum Entwurf einer historischen Interpretationsforschung $\quad 28$

Manuel Bärtsch >Interpretation‘. Beethovens Sonate A-Dur op. IOI in der Sicht von Eugen d'Albert und Frederic Lamond

Sebastian Bausch Klavierrollen als Interpretationsdokumente. Ein Erfahrungsbericht als Leitfaden für Einsteiger $\quad 7 \mathrm{I}$

Camilla Köhnken Beethoven-Auslegung zwischen Liszts »Deklamationsstil« und Bülows »Vivisektionsversuchen«. Auf den Spuren Liszt'scher Interpretationsideale in Hans von Bülows instruktiver Edition der Klaviersonaten Beethovens $\quad 92$

Neal Peres Da Costa Carl Reinecke's Performance of his Arrangement of the Second Movement from Mozart's Piano Concerto K. 488. Some Thoughts on Style and the Hidden Messages in Musical Notation

II4

Carolina Estrada Bascuñana Enrique Granados's Performance Style.

Visualising the Audible Evidence I5O

Lukas Näf Tempogestaltung in Weberns Sinfonie op. 2I I80

INTERPRETATION - AUFFÜHRUNGSGESCHICHTE

Christoph Moor "Ein so erklärtes Lieblingsstück der hiesigen Kunstfreunde«. Die Rezeptionsgeschichte der Jupiter-Sinfonie in Beethovens Wien

Luisa Klaus Objektive Bruckner-Interpretation? Zur Aufführung der Trio-Entwürfe für die Neunte Sinfonie I940 205

Chris Walton Von innen und von außen. Beethovens Neunte Sinfonie und die $>$ Wagner'sche< Dirigiertradition $\quad 2 \mathrm{I} 8$

Lena-Lisa Wüstendörfer Streit um Fidelio. Gustav Mahler und Felix Weingartner im Disput um Werktreue $\quad 238$

INTERMEZZO

Robert Levin Turning Point to Musical Modernity. Beethoven as Executor of the Legacy of C. P. E. Bach. Concert Lecture 249

INTERPRETATION - INSTRUMENTE, ANALYSE, EDITION

Martin Skamletz «Man hat diese Erweiterung des Tonumfanges seit ein paar Jahren an den Tasteninstrumenten sehr weit getrieben.« Der Umgang mit Grenzen beim späten Mozart und beim frühen Beethoven $\quad 263$ 
Stephan Zirwes Analyse und Interpretation. Adolph Bernhard Marx' Beethoven-Analysen 29I

Michael Ladenburger Was können wir aus Originalhandschriften von Beethoven für eine angemessene Interpretation lernen? 30I

Federica Rovelli Die Skizzenbuch-Ausgaben und ihre mögliche digitale Zukunft 3 I7

Johannes Gebauer Interpretationspraktische Stemmatik. Philologische Methoden in der Interpretationsforschung am Beispiel annotierter Notenausgaben von Rodes 24 Capricen und Beethovens Violinkonzert

John Rink Chopin Copying Chopin 349

Tomasz Herbut Alexander Goldenweiser und Beethovens Sonate op. IIo- eine Spurensuche 366

INTERPRETATION - KREATIVE ANEIGNUNG

Thomas Gartmann Beethoven als sein eigener Interpret. Gedanken zur Bearbeitung der Klaviersonate op. I4/ז für Streichquartett

Ivo Haag Die Sinfonien von Johannes Brahms - (auch) Klaviermusik?

Michael Lehner Das Orchester auf dem Klavier. Welte-Klavierrollen von Gustav Mahler und Richard Strauss als interpretationsanalytische Quellen

Roger Allen “That Is What Music Really Is". Richard Wagner's Reception of Beethoven's Piano Sonata in A Major Op. IOI $43 \mathrm{I}$

Daniel Allenbach Eine heroische< Neunte? Dmitri Schostakowitschs Neunte Sinfonie im Vergleich mit Ludwig van Beethoenvs Sinfonien Nr.3 und 9 44I

Simeon Thompson Beethoven und der Zweite Weltkrieg in der künstlerischen Reflexion der Nachkriegszeit. Stanley Kubricks A Clockwork Orange und Rolf Liebermanns Leonore 40/45 456

Michelle Ziegler Rettungsversuch im Jubiläumsjahr. Mauricio Kagels Aufarbeitung der Beethoven-Rezeption in der Ludwig van-Werkgruppe (I970) 465

Leo Dick Über den späten Beethoven zur >Postidentität،. Die Suche nach liminalen Räumen im gegenwärtigen Musiktheater am Beispiel von Matthias Rebstocks Berliner Produktion Büro für postidentisches Leben $\quad 476$

Elizabeth Waterhouse Choreographic Re-mix. William Forsythe's Trio (I996) and Beethoven's String Quartet No. I5 in a Minor Op. I32 487

László Stachó "Gradus ad Parnassum".

The Purgatory of Instrumental Technique

Namen-, Werk- und Ortsregister 522

Die Autorinnen und Autoren der Beiträge 


\section{Rund um BeEthoven \\ Interpretationsforschung heute • \\ Herausgegeben von Thomas}

Gartmann und Daniel Allenbach 


\section{MUSIKFORSCHUNG DER Hochschule der KÜnste Bern Herausgegeben von Martin Skamletz und Thomas Gartmann Band 14}


0 Dieses Buch ist in gedruckter Form im Dezember 2019 in erster Auflage in der Edition Argus in Schliengen/Markgräflerland erschienen. Gestaltet und gesetzt wurde es im Verlag aus der Seria und der SeriaSans, die von Martin Majoor im Jahre 2000 gezeichnet wurden. Gedruckt wurde es auf Eos, einem holzfreien, säurefreien, chlorfreien und alterungsbeständigen Werkdruckpapier der Papierfabrik Salzer im niederösterreichischen Sankt Pölten. Das Vorsatzpapier Caribic cherry wurde von Igepa in Hamburg geliefert. Rives Tradition, ein Recyclingpapier mit leichter Filznarbung, das für den Bezug des Umschlags verwendet wurde, stellt die Papierfabrik Arjo Wiggins in Issy-les-Moulineaux bei Paris her. Das Kapitalband mit rot-schwarzer Raupe lieferte die Firma Dr. Günther Kast aus Sonthofen im Oberallgäu, die auf technische Gewebe und Spezialfasererzeugnisse spezialisiert ist. Gedruckt und gebunden wurde das Buch von der Firma Bookstation im bayerischen Anzing. Im Internet finden Sie Informationen über das gesamte Verlagsprogramm unter www.editionargus.de, zum Institut Interpretation der Hochschule der Künste Bern unter www.hkb.bfh.ch/interpretation und www.hkb-interpretation.ch. Die Deutsche Nationalbibliothek verzeichnet diese Publikation in der Deutschen Nationalbibliografie; detaillierte bibliografische Daten sind im Internet über www.dnb.de abrufbar. (c) der zeitgleich erschienenen digitalen Version: die Autorinnen und Autoren, 20I9. Dieses Werk ist lizenziert unter einer Creative Commons Namensnennung-Nicht kommerziell 4.0 International Lizenz (CC BY-NC 4.o). DoI: https://doi.org/I0.26045/kp64-6I78 ISBN 978-3-93I264-94-9 